\title{
Stretchable, Transparent, Ionic Conductors
}

\section{Citation}

Keplinger, Christoph, Jeong Yun Sun, Choon Chiang Foo, Philipp Josef Michael Rothemund, George M. Whitesides, and Zhigang Suo. 2013. Stretchable, transparent, ionic conductors.

Science 341(6149): 984-987.

\section{Published Version}

doi:10.1126/science. 1240228

\section{Permanent link}

http://nrs.harvard.edu/urn-3:HUL.InstRepos:11084802

\section{Terms of Use}

This article was downloaded from Harvard University's DASH repository, and is made available under the terms and conditions applicable to Other Posted Material, as set forth at http:// nrs.harvard.edu/urn-3:HUL.InstRepos:dash.current.terms-of-use\#LAA

\section{Share Your Story}

The Harvard community has made this article openly available.

Please share how this access benefits you. Submit a story.

Accessibility 


\title{
Stretchable, Transparent, Ionic Conductors \\ Christoph Keplinger ${ }^{1,2,3^{*}}$, Jeong-Yun Sun ${ }^{1,2^{*}}$, Choon Chiang Foo ${ }^{1,2,4}$, George M. Whitesides ${ }^{2,3,5^{\dagger}}$, and Zhigang Suo $^{1,2^{\dagger}}$
}

\begin{abstract}
This paper shows that stretchable, transparent, ionic conductors (STICs) enable the fabrication of devices with new attributes. In demonstrations of performance, a transparent actuator generates an area strain of $167 \%$ at $18 \mathrm{kV}$, and a transparent loudspeaker produces sound over the entire audible range. These devices achieve high-voltage and high-frequency electromechanical transduction without electrochemical reaction. The ionic conductors exhibit transmittance of $99.99 \%$ at $550 \mathrm{~nm}$, linear strain beyond 500\%, and sheet resistance below 200 $\Omega /$ sq. At large stretch and high transmittance, the ionic conductors have lower electrical resistance than all existing stretchable and transparent electronic conductors. STICs offer new opportunities for scientific exploration, and for applications.
\end{abstract}

Keywords: Stretchable ionics, ionic conductors, stretchable conductors, transparent conductors, transparent actuators, transparent loudspeakers.

${ }^{1}$ School of Engineering and Applied Sciences, Harvard University, Cambridge, MA 02138, USA. ${ }^{2}$ Kavli Institute for Bionano Science and Technology, Harvard University, Cambridge MA 02138, USA. 3Department of Chemistry and Chemical Biology, Harvard University, Cambridge, MA 02138, USA. 4Institute of High Performance Computing, 1 Fusionopolis Way, Singapore 138632 5Wyss Institute for Biologically Inspired Engineering, Harvard University, Cambridge MA 02138, USA.

*These authors contributed equally to this work.

† To whom correspondence should be addressed:

GWhitesides@gmwgroup.harvard.edu, suo@seas.harvard.edu 
The past century has seen the rise of electronics-engineered devices in which electrons carry electrical charge. The successful rise of electronics, however, has overshadowed another success with a much longer history-ionics, Nature's solution to charge transport, based on ions and water. Combining these parallel worlds-the engineered (using electrons) and the natural (using ions)-is creating a hybrid field: bioelectronics. Examples of applications include electrode arrays, where the electronics of medical instruments meet the ionics of tissues and cells (1), and brain-machine interfaces, through which cortical ionic impulses control prosthetic $\operatorname{arms}(2)$.

A current challenge to bioelectronics is to bridge a common gap-in mechanics-between electronics and ionics. Electronic systems are usually made of hard materials, while tissues and cells are soft. The field of "stretchable electronics" is developing rapidly, and promises to make electronics conformal to the skin, heart and brain $(3,4)$. Stretchable conductors are also needed in non-biomedical applications, such as electromechanical transduction (5) and solar energy conversion (6). Existing stretchable conductors are mostly electronic conductors, including carbon grease (7), micro-cracked gold films (8), serpentine-shaped metallic wires (3), carbon nanotubes $(9,10)$, graphene sheets $(11,12)$, and silver nanowires $(13-15)$. Stretchable devices have also been made using corona discharge (16), liquid metals (17), and saline solutions (18); these conductors, however, are not solid, and their uses are limited. Attributes other than conductivity and stretchability are, of course, also important in specific applications. For example, for some applications stretchable conductors must operate at high frequencies and high voltages (7), remain conductive under repeated expansion in area beyond 1000\% (19), be biocompatible (5), and be transparent (9-15).

While electronic conductors struggle to meet these demands, ionic conductors meet most of them readily. Many ionic conductors, such as hydrogels (20) and gels swollen with ionic liquids (21), take a solid form, and are stretchable and transparent. Ionic conductors constitute a 
large class of materials; their diversity enables them to meet requirements in addition to conductivity, stretchability and transparency. Many ionic hydrogels, for example, are biocompatible and conformal to tissues and cells down to the molecular scale (22). This paper demonstrates, surprisingly, that ionic conductors can even be used to fabricate devices that operate at high voltage and high frequency. We build an actuator achieving large deformation at high voltage, and a loudspeaker producing sound across the entire audible range. These devices are essentially perfectly transparent to light across the entire visible range. We study the fundamental limits of such electromechanical transduction by a combination of experiment and theory. We derive the conditions under which the ionic conductors enable electromechanical transduction without electrochemical reaction. The strain of actuation is not limited by the elasticity of the soft ionic conductors, but by electromechanical instability. The frequency of actuation is not limited by electrical resistance, but by mechanical inertia. At large stretch and high transmittance, elastomeric ionic conductors have lower electrical resistance than all existing stretchable and transparent electronic conductors.

Stretchable, transparent, ionic conductors (STICs) enable many devices, which we call "stretchable ionics" for brevity. One basic design of stretchable ionics places two electrodes (electronic conductors), an electrolyte (ionic conductor) and a dielectric (insulator) in series (Fig. 1A). The electrode/electrolyte interface forms an electrical double layer (Fig. 1B). For some combinations of the electrode and electrolyte, if the voltage across the interface is within a certain range $(\sim 1 \mathrm{~V})$, the interface is ideally polarizable-that is, electrons and ions do not cross the interface, no electrochemical reaction occurs, and the electrical double layer behaves like a capacitor (Fig. 1C). The electrolyte and the dielectric in series establish capacitive coupling between the electrical signals carried by the two electrodes, and transport power with alternating current. 
Provided the electrode/electrolyte interface is ideally polarizable, and the dielectric is a perfect insulator, at equilibrium the voltage $V$ applied between the two electrodes is carried entirely by the electrical double layer and the dielectric, $V=V_{E D L}+V_{D}$. Charges in the electrode and in the electrolyte are separated over nanometers; this small separation leads to a large capacitance, on the order of $c_{E D L} \sim 10^{-1} \mathrm{~F} / \mathrm{m}^{2}$ (21). By contrast, charges on the two faces of the dielectric are separated over its thickness (about millimeters in our experiments); this large separation leads to a small capacitance, on the order of $c_{D} \sim 10^{-8} \mathrm{~F} / \mathrm{m}^{2}$. In response to the applied voltage, the two capacitors add the same amount of charge, so that $c_{D} A_{D} V_{D}=c_{E D L} A_{E D L} V_{E D L}$, where $A_{E D L}$ is the area of the electrical double layer, and $A_{D}$ is the area of the dielectric. In our experiments, $A_{E D L} / A_{D} \sim 0.1$; the large ratio $c_{E D L} / c_{D} \sim 10^{7}$ ensures that the voltage across the electrical double layer $V_{E D L}$ is well below $1 \mathrm{~V}$ when the voltage across the dielectric is on the order of $V_{D} \sim 10 \mathrm{kV}$. A small $V_{E D L}$ prevents electrochemical reaction, while a large $V_{D}$ enables electromechanical transduction. The electrode/electrolyte interface may form thin layers of reaction products, so long as they are stable under a small voltage. The applied voltage $V$ is limited not by electrochemical reaction at the electrode/electrolyte interface, but by electrical breakdown in the dielectric (Supplementary Materials).

To demonstrate the remarkable properties of STICs, we build a transparent, high-speed, large-strain actuator using a design which we call a "layered electrolytic and dielectric elastomer" (LEADER). A membrane of a dielectric elastomer is sandwiched between two membranes of the electrolytic elastomer (Fig. 2A and fig. S1). The electrolytes and the dielectric are stretchable and transparent, but the electrodes need not be. So long as the electrodes are placed outside the active area of the device, the actuator is stretchable and transparent. When a voltage is applied between the electrodes, ions of different charge $7 / 15 / 13$ 
polarities collect on the two electrolyte/dielectric interfaces; the oppositely charged interfaces attract each other, and cause the sandwich to reduce its thickness and enlarge its area (Fig. 2B).

We demonstrate this design using 1-mm-thick $3 \mathrm{M}^{\mathrm{TM}} \mathrm{VHB}^{\mathrm{TM}} 4910$ as the dielectric, and 100- $\mu$ m-thick polyacrylamide hydrogel containing $2.74 \mathrm{M} \mathrm{NaCl}$ as the electrolyte. To compare the performance of the ionic conductor to an electronic conductor, we use the electrolytic elastomer to replace carbon grease in an existing design of an electrostatic actuator (7). We stack three layers of the VHB together, stretch them radially to three times their initial radius, and fix them to a circular acrylic frame (Figs. $2 \mathrm{C}$ and $2 \mathrm{D}$ ). We then sandwich the dielectric stack between two layers of heart-shaped hydrogels, which are linked through thin hydrogel lines to copper electrodes placed on the frame. When a voltage is applied and removed, the heart expands and contracts (movie S1). The beating heart is transparent to all colors (Figs. 2E and $2 \mathrm{~F})$.

We model the electromechanical transduction of the actuators, and compare the theory with experiments using the dielectric sandwiched between layers of hydrogels of circular shape (fig. S2). An area strain of $167 \%$ is achieved at $18 \mathrm{kV}$ (Fig. $2 \mathrm{G}$ ). This voltage-induced strain is limited by electromechanical instability, and the soft hydrogels do not constrain the dielectric (fig. S3). The area strain of our actuator reduces as the frequency of applied voltage increases, and becomes vanishingly small at a frequency on the order of $10^{3} \mathrm{~Hz}$ (Fig. 2H). These characteristics of the actuator using the hydrogel are comparable to those of actuators using carbon grease (7), but the carbon grease is an opaque electronic conductor, while the hydrogel is a transparent ionic conductor.

Our analysis shows that the frequency of actuation is not limited by electrical resistance, but by mechanical inertia (Supplementary Materials). The time to charge the device, the RC delay, scales as $\tau_{\mathrm{RC}} \sim c_{D} A_{D} R$, where $R$ is the sheet resistance of the ionic conductor. For 
representative values $c_{D}=10^{-8} \mathrm{~F} / \mathrm{m}^{2}, A_{D}=10^{-2} \mathrm{~m}^{2}$, and $R=10^{2} \Omega$, we estimate that $\tau_{\mathrm{RC}} \sim 10^{-8} \mathrm{~s}$, corresponding to a frequency much higher than the observed limiting frequency of actuation. The fundamental resonance sets a time $\tau_{\text {inertia }} \sim \sqrt{A_{D} \rho / Y}$, where $\rho$ is the mass density, and $Y$ the elastic modulus. For representative values $\rho=10^{3} \mathrm{~kg} / \mathrm{m}^{3}$ and $Y=10^{5} \mathrm{~N} / \mathrm{m}^{2}$, we estimate that $\tau_{\text {inertia }} \sim 10^{-3} \mathrm{~s}$; this value is consistent with the observed limiting frequency of actuation.

To demonstrate that the STICs can enable electromechanical transduction much beyond the fundamental resonance, we build a transparent loudspeaker that produces sound from 20 $\mathrm{Hz}$ to $20 \mathrm{kHz}-$ that is, across the entire audible range. The fabrication process is similar to that of the actuator. The loudspeaker is placed in front of a laptop, which plays music videos (Figs. 3A, 3B and fig. S4). The screen of the laptop is clearly visible through the loudspeaker. The sound tracks of the videos are fed to the loudspeaker as analog voltage signals, from the audio output of the laptop, through a high-voltage amplifier. A video of the transparent loudspeaker and the sound produced are recorded with a webcam at a distance of $15 \mathrm{~cm}$ (movie S2).

We study the fidelity of the sound reproduction by feeding the loudspeaker with a $20 \mathrm{~s}$ test signal of constant amplitude and a linear sine sweep from $20 \mathrm{~Hz}$ to $20 \mathrm{kHz}$ (Figs. $3 \mathrm{C}$ and ${ }_{3} \mathrm{D}$ ). The sound generated by the loudspeaker is recorded by the webcam (movie $\mathrm{S}_{3}$ ). In the first few seconds, the amplitude of the recorded sound is large (Fig. 3E). This interval reflects the resonance of the frame of the loudspeaker, which is not optimized to suppress vibration. The amplitude varies only slightly over the remainder of the recording. The spectrogram of the recorded sound displays the successful reproduction of the main signal of the original test sound throughout the audible frequency range (Fig. 3F). In the lower part of the tested frequency range, vibrations of the frame and the membrane are visible (movie $\mathrm{S} 4$ ).

At large stretch and high transparency, the ionic conductors have lower resistance than existing stretchable and transparent electronic conductors, such as silver nanowires (AgNWs), 
single-wall carbon nanotubes (SWNTs), graphene, and indium tin oxide (ITO). An 11-mmthick hydrogel containing $5.48 \mathrm{M} \mathrm{NaCl}$ shows $98.9 \%$ average transmittance in the visible range (Fig. 4A), corresponding to a transmittance of $99.99 \%$ for a $100-\mu \mathrm{m}$-thick hydrogel used in constructing actuators and loudspeakers. The resistivity of the hydrogel is almost identical to that of water containing the same concentration of $\mathrm{NaCl}$ when the hydrogel is not stretched, and increases when the hydrogel is stretched (Fig. 4B). Among all electrical conductors, these hydrogels show the highest transmittance (Fig. 4C). For a conductor whose resistivity is independent of deformation, the resistance of the conductor increases with stretch as $R / R_{\mathrm{o}}=\lambda^{2}$, where $R_{\mathrm{o}}$ is the resistance before the conductor is stretched, and $R$ is the resistance after the conductor is stretched $\lambda$ times its initial length. This prediction closely approximates the measured resistance-stretch curve for the elastomeric ionic hydrogel (Fig. 4D). By contrast, when SWNTs on VHB are stretched, the resistance increases faster by orders of magnitude than the prediction of the square law, indicating that the resistivity of the SWNTs is increased greatly by the stretch.

Our design of layered electrolytic and dielectric elastomers should be compared with existing actuators in which ionic conduction plays essential roles. Examples include actuators made of carbon nanotubes and conducting polymers in electrolytes $(23,24)$, actuators made of ionic polymer-metal composites (25), and resistive strain sensors made of elastomeric ionic hydrogels (26). Our design introduces a dielectric in series with an electrolyte, and the small capacitance of the dielectric enables high-speed and large-strain actuation. In a design of electrowetting devices, electrolytes and dielectrics are in series, but the electrolytes are liquids, and the dielectrics do not deform (27).

Stretchable ionics offer new opportunities for designers of soft machines. Hydrogels may well be the STICs of choice to demonstrate conceptual designs, and to fabricate devices that require biocompatibility. The diversity of ionic conductors, however, creates a large pool of candidates for specific applications. Hydrogels dry as water evaporates; gels with nonvolatile 7/15/13 
solvents should be explored as possible replacements, as should ionic liquids and gels swollen with ionic liquids (21). Dielectric elastomers have been paired with electronic conductors to make transducers in robotics, bioelectronics and energy harvesting (28). Pairing dielectric elastomers with STICs will significantly expand the scope of applications, for example, leading to devices such as tunable optics and localized haptics with transparent ionic conductors placed in the path of the light. The ultrahigh transparency and compliance of the ionic conductors will enable transparent devices of multilayered electrolytic and dielectric elastomers (multiLEADERs), operating at lower voltage. To scale up the active area of a device, one can enlarge the electrode/electrolyte interface by using porous electrodes. Loudspeakers with some transparency have been made using electronic conductors, such as graphene sheets (29) and carbon nanotubes (30). We have shown that ionic conductors allow the fabrication of loudspeakers having almost perfect transparency. Transparent loudspeakers might also, for example, be attached to windows to achieve active noise cancelation (30). The LEADER also works for applications that require low voltage and low frequency. When stretched mechanically, the LEADER increases area and reduces thickness, so that its capacitance increases. This characteristic will enable transparent capacitive strain sensors conformal to soft tissues and operating at low voltage.

There is only one kind of electron, but there are many kinds of ions. This diversity will enable ionic conductors to be designed for many applications. Life uses primarily ions-rather than electrons-to carry electrical charge. In creating biomedical and engineering devices, it is well to consider the opportunity: the hard and the soft do not necessarily have to meet through electronic conductors; they may as well meet through ionic conductors.

Acknowledgement This work is supported by the NSF MRSEC (DMR-0820484), and by the ARO (W911NF-09-1-0476). We acknowledge DOE award \# ER45852 for salary support of C.K.; C.C.F. acknowledges support from A*STAR AGS (PDF). 


\section{References and Notes}

1. M. E. Spira, A. Hai, Multi-electrode array technologies for neuroscience and cardiology. Nature Nanotechnol. 8, 83-94 (2013).

2. M. Velliste, S. Perel, M. C. Spalding, A. S. Whitford, A. B. Schwartz, Cortical control of a prosthetic arm for self-feeding. Nature 453, 1098-1101 (2008).

3. D.-H. Kim et al., Epidermal electronics. Science 333, 838-843 (2011).

4. T. Someya, Ed., Stretchable Electronics (Wiley-VCH, Weinheim, Germany, 2013).

5. S. Rosset, H.R. Shea, Flexible and stretchable electrodes for dielectric elastomer actuators, Appl. Phys. A 110, 281-307 (2013).

6. D.J. Lipomi, Z. Bao, Stretchable, elastic materials and devices for solar energy conversion. Energy Environ. Sci. 4, 3314-3328 (2011).

7. R. Pelrine, R. Kornbluh, Q. Pei, J. Joseph, High-speed electrically actuated elastomers with strain greater than 100\%. Science $\mathbf{2 8 7}, 836-839$ (2000).

8. S. P. Lacour, S. Wagner, Z. Huang, Z. Suo, Stretchable gold conductors on elastomeric substrates. Appl. Phys. Lett. 82, 2404-2406 (2003).

9. L. Hu, W. Yuan, P. Brochu, G. Gruner, Q. Pei, Highly stretchable, conductive, and transparent nanotube thin films. Appl. Phys. Lett. 94, 161108 (2009).

10. V. Scardaci, R. Coull, J.N. Coleman, Very thin transparent, conductive carbon nanotube films on flexible substrates. Appl. Phys. Lett. 97, 023114 (2010).

11. K.S. Kim et al., Large-scale pattern growth of graphene films for stretchable transparent electrodes. Nature 457, 706-710 (2009).

12. S. De et al., Flexible, transparent, conducting films of randomly stacked graphene from surfactant-stabilized, oxide-free graphene dispersions. Small 6, 458-464 (2010).

13. S. De et al., Silver nanowire networks as flexible, transparent, conducting films: extremely high dc to optical conductivity ratios. ACS Nano 3, 1767-1774 (2009).

14. Z. Yu et al., Highly flexible silver nanowire electrodes for shape-memory polymer lightemitting diodes. Adv. Mater. 23, 664-668 (2011).

15. W. Hu et al., Intrinsically stretchable transparent electrodes based on silver-nanowirecrosslinked-polyacrylate composites. Nanotechnology 23, 344002 (2012).

16. C. Keplinger, M. Kaltenbrunner, N. Arnold, S. Bauer, Röntgen's electrode-free elastomer actuators without electromechanical pull-in instability. Proc. Natl. Acad. Sci. U.S.A. 107, 4505-4510 (2010).

17. M.D. Dickey et al., Eutectic Gallium-Indium (EGaIn): a liquid metal alloy for the 
formation of stable structures in microchannels at room temperature. Adv. Funct. Mater. 18, 1097-1104 (2008).

18. Q. Zhao et al., Electric-field-tuned color in photonic crystal elastomers. Appl. Phys. Lett. 100, 101902 (2012).

19. C. Keplinger, T. Li, R. Baumgartner, Z. Suo, S. Bauer, Harnessing snap-through instability in soft dielectrics to achieve giant voltage-triggered deformation. Soft Matter 8, 285-288 (2012).

20. J.Y. Sun et al., Highly stretchable and tough hydrogels. Nature 489, 133-136 (2012).

21. K.H. Lee et al., "Cut and stick" rubbery ion gels as high capacitance gate dielectrics. Adv. Mater. 24, 4457-4462 (2012).

22. K. Y. Lee and D.J. Mooney, Hydrogels for tissue engineering. Chem. Rev. 101, 18691879 (2001).

23. R. H. Baughman et al., Carbon nanotube actuators. Science 284, 1340-1344 (1999).

24. E.W.H. Jager, E. Smela, O. Ingana, Microfabricating conjugated polymer actuators. Science 290, 1540-1545 (2000).

25. M. Shahinpoor, Y. Bar-Cohen, J.O. Simpson, J. Smith, Ionic polymer-metal composites as biomimetic sensors, actuators and artificial muscles - a review. Smart Mater. Struct. 7, 1-27 (1998).

26. P. Manandhar, P.D. Calvert, J.R. Buck, Elastomeric ionic hydrogel sensor for large strains. IEEE Sens. J. 12, 2052-2061 (2012).

27. C. Quilliet, B. Berge, Electrowetting: a recent outbreak. Curr. Opin. Colloid \& Interface Sci. 6, 34-39 (2001).

28. F. Carpi, S. Bauer, D. De Rossi, Stretching dielectric elastomer performance. Science 330, 1759-1761 (2010).

29. S.C. Xu et al., Flexible and transparent graphene-based loudspeakers. Appl. Phys. Lett. 102, 151902 (2013).

30. X. Yu, R. Rajamani, K.A. Stelson, T. Cui, Carbon nanotube-based transparent thin film acoustic actuators and sensors. Sensors Actuators A 132, 626-631 (2006). 
A


Fig.1. A basic design of stretchable ionics. (A) An electrolyte and a dielectric are in series. When they are elastomeric, the device is solid and stretchable. When a voltage $V$ is applied between the two electrodes, the voltage across the electrode/electrolyte interface is much smaller than the voltage across the dielectric, $V_{E D L}<<V_{D}$. (B) The electrode/electrolyte interface forms an electrical double layer. (C) When the voltage across the interface is within a certain range, electrons and ions do not cross the interface, no electrochemical reaction occurs, and the electrical double layer behaves like a capacitor. 
A

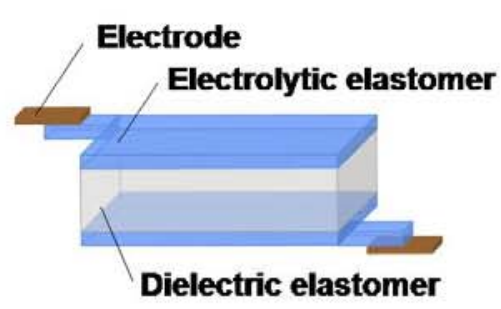

Voltage off

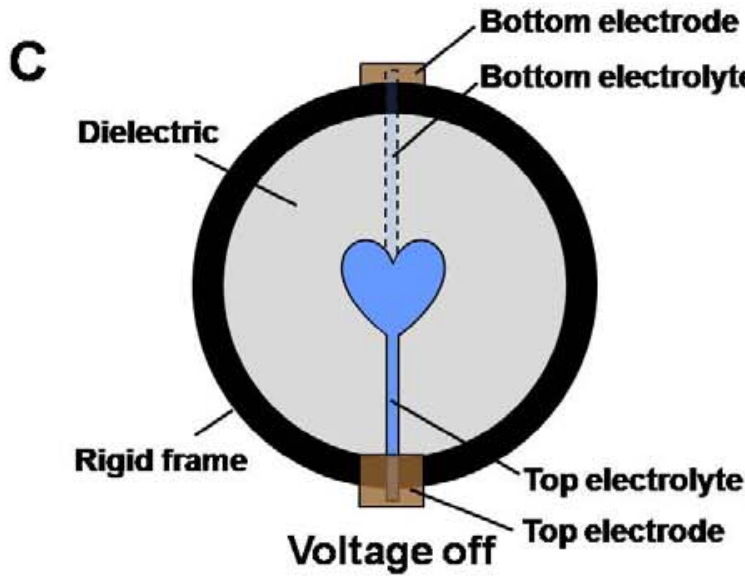

E

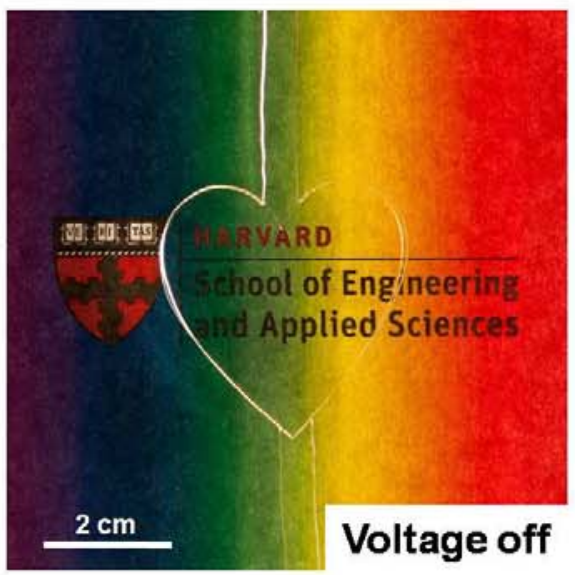

G

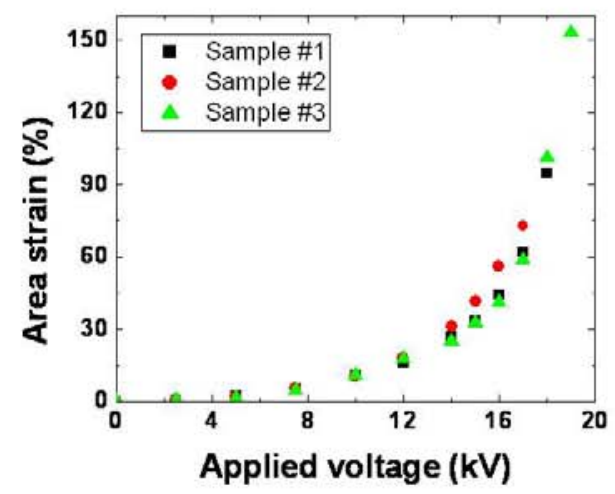

B

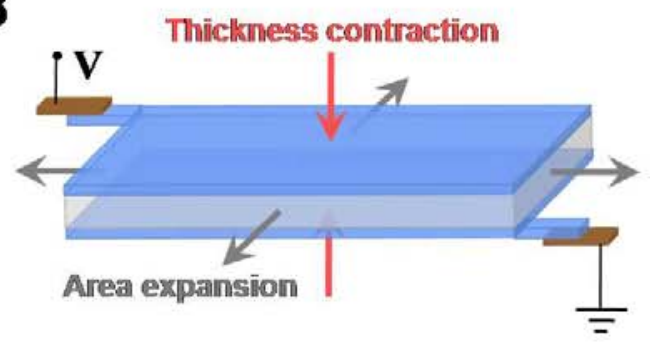

Voltage on

D

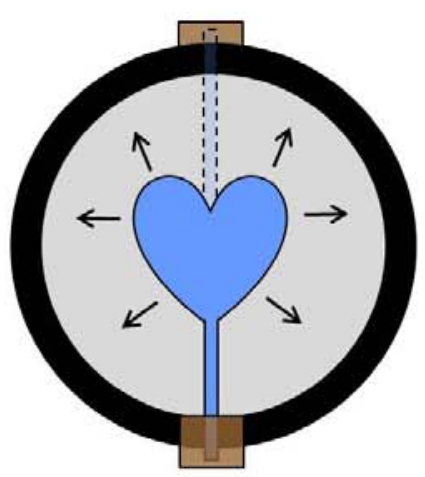

Voltage on

F

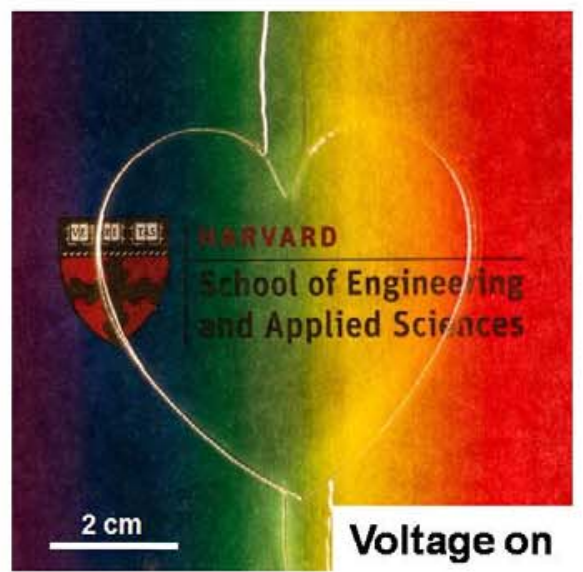

H

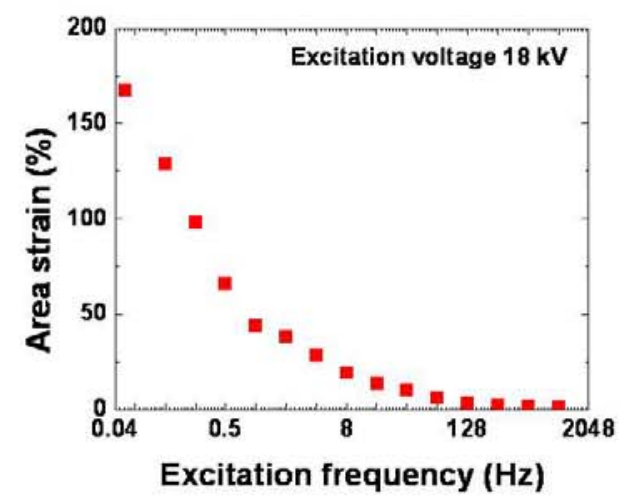


Fig. 2. Transparent actuator capable of fast voltage-induced deformation. (A) A dielectric elastomer is sandwiched between two layers of an electrolytic elastomer. Both the dielectric and electrolyte are transparent and stretchable, and the device is transparent if the electrodes are placed outside the active area of the device. (B) Subject to voltage, the two layers of the electrolyte spread ions of opposite signs on the two sides of the dielectric, causing the sandwich to reduce thickness and expand area. (C) A specific design of the actuator with the electrolyte in the shape of a heart. (D) The heart expands when the voltage is applied. (E) and (F) The actuator is transparent to all colors. The area strain is measured as a function of voltage (G), and as a function of the frequency $(\mathbf{H})$, using an actuator with electrolytes of circular shape. 

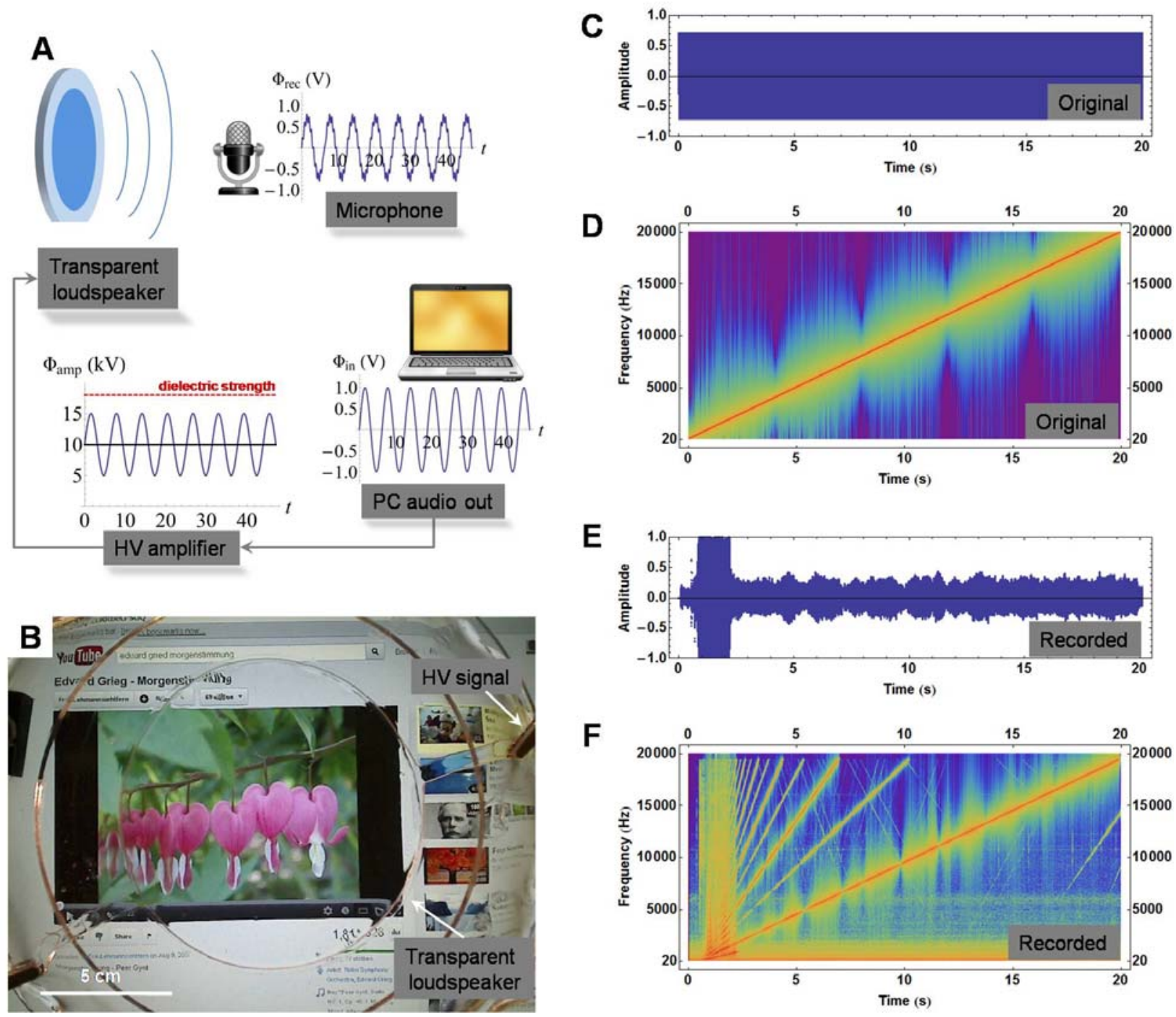

Fig. 3. Transparent loudspeaker capable of producing sound across the entire audible range. (A) The voltage signal from the audio output of a laptop is fed through a high voltage amplifier to the loudspeaker. The loudspeaker transforms the voltage signal into sound, which is recorded with a microphone. (B) The screen of the laptop is clearly visible through the loudspeaker. Amplitude (C) and spectrogram (D) of a test signal with constant amplitude and a linear sine sweep of frequency from $20 \mathrm{~Hz}$ to $20 \mathrm{kHz}$. The colors correspond to the intensities of frequency, with warmer colors indicating higher intensity. Amplitude (E) and spectrogram (F) of the sound generated by the loudspeaker. 

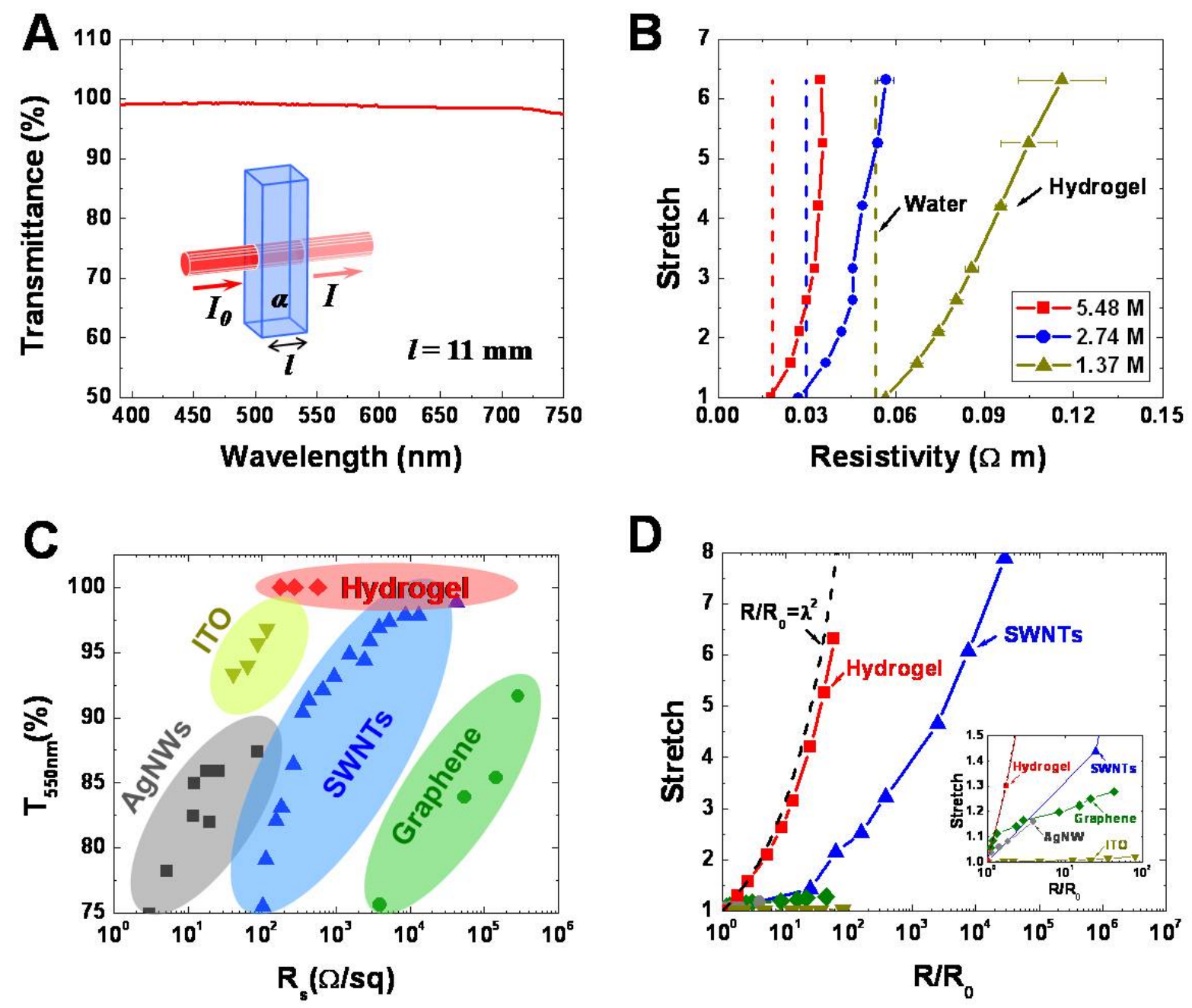

Fig. 4. Performance of ionic and electronic conductors. (A) A $11 \mathrm{~mm}$ thick polyacrylamide hydrogel containing $5.48 \mathrm{M} \mathrm{NaCl}$ shows $98.9 \%$ average transmittance in the visible range. (B) Electrical resistivities of hydrogels of several concentrations of $\mathrm{NaCl}$ were measured as functions of stretch, and compared with the resistivities of water containing the same concentrations of $\mathrm{NaCl}$. (C) Transmittance (at $550 \mathrm{~nm}$ ) is plotted against sheet resistance for ITO (14), AgNWs (13), SWNTs (10), Graphene (12) and hydrogels (100 $\mu \mathrm{m}$ thickness, this work). (D) Stretch is plotted against normalized resistance for ITO (15), AgNWs (14), Graphene (11), SWNTs (9) and hydrogel (this work). 